IJRMMS-D-16-00084 - edited and approved by editor

\title{
Effects of Small-Amplitude Periodic Topography on Combined Stresses due to Gravity and Tectonics
}

\author{
Stephen J. Martel \\ University of Hawaii, Department of Geology and Geophysics
}

Corresponding author:

Stephen J. Martel

Department of Geology and Geophysics

University of Hawaii, 1680 East-West Road

Honolulu, HI, 96822

e-mail: $\underline{\text { smartel@hawaii.edu }}$

tel: $1-808-856-7797$ 


\begin{abstract}
Topographic perturbations of gravitational body forces and horizontal tectonic stresses can be substantial, non-intuitive, and important in terms of subsurface engineering and rock fracture near the surface of the Earth. For (co)sinusoidal topography where the amplitude $(A)$ is small relative to the wavelength $(L)$, adjustments to published plane strain (two-dimensional) approximate elastic solutions for stresses in uniform, isotropic rock allow effects of gravity and a uniform regional horizontal stress $(T)$ to be distinguished. These first-order solutions contain a characteristic stress and three geometric terms, one that varies linearly with elevation, one that decays exponentially with depth, and a (co)sinusoidal term; elastic moduli do not enter the solutions. The first-order solutions are useful approximations for $A / L<0.04$. Both gravity and regional compression yield a compression parallel to the surface at ridge crests. Gravity, by itself, causes a localized horizontal tension below valley bottoms. Regional horizontal compression, by itself, contributes a localized vertical tension beneath ridge crests. If $T$ is about an order of magnitude less compressive than $\rho g A$, where $\rho$ is rock density, and $g$ is gravitational acceleration, then effects of gravity dominate effects of the regional compression near the topographic surface. These conditions promote opening of vertical fractures at valley bottoms. Conversely, if $T$ is about an order of magnitude more compressive than $\rho g A$, then effects of regional compression dominate the effects of gravity near the topographic surface. These conditions promote the opening of sheeting joints, macroscopic fractures that open near to and essentially parallel to the topographic surface.
\end{abstract}

$\underline{\text { Key words (maximum of } 6 \text { ) }}$

Topography

Stresses

Fracture

Sheeting joints 


\section{Introduction}

Effects of topography on stresses in rock in the shallow subsurface have drawn research interest for myriad reasons for decades. ${ }^{1-14}$ For example, topographic stress perturbations affect tunnel safety, ${ }^{15}$ slope stability, ${ }^{16}$ volcanic processes, ${ }^{17-19}$ and groundwater flow. ${ }^{20,21}$ Topographic stress perturbations are likely to be critical in the formation of hydraulic fractures in certain areas, as well as in the formation of sheeting joints, opening mode fractures at shallow depths that essentially parallel the topographic surface..$^{22,23}$ In spite of the widely recognized importance of topography on the near-surface stress field and many advances in numerical modeling, considerable room remains for understanding basic factors of how topography perturbs ambient stresses in the shallow subsurface.

Topographic perturbations of stresses arising from gravity and tectonic loads have drawn particular attention. Although the topographic surface of the Earth clearly is three-dimensional, four two-dimensional theoretical treatments currently stand out for illuminating how topography perturbs gravitational and tectonic stresses. Holzhausen ${ }^{2}$ and Savage et $\mathrm{al}^{5}$ formulated solutions for topographic perturbations of the stress field arising from horizontal tectonic stresses. Savage and Swolfs ${ }^{6}$ and Haneberg ${ }^{24}$ developed corresponding solutions for the topographic perturbations of gravitational body forces. Holzhausen ${ }^{2}$ and Haneberg ${ }^{24}$ provided first-order approximate solutions for small amplitude periodic topography, whereas Savage et $\mathrm{al}^{5}$ and Savage and Swolfs ${ }^{6}$ derived exact solutions for bell-shaped topography. All of these solutions treat rock as a uniform, isotropic, isothermal linear elastic material. They provide benchmarks for numerical solutions, are relatively simple, and can be used to isolate critical length scales and characteristic stresses. Somewhat surprisingly, however, the relative contributions of gravitational and tectonic stresses to the total stress field do not seem to have been considered.

This contribution focuses exclusively on two-dimensional theoretical considerations of how periodic topography of small amplitude perturbs gravitational stresses and regional horizontal stresses. The amplitude $(A)$ is considered to be small if it is small relative to the wavelength $(L)$. No closed form exact solutions have been discovered for these problems. The two-dimensional, plane strain, linear elastic solutions considered here are for a vertical cross section perpendicular to the horizontal axis of periodic ridges and valleys. The displacements perpendicular to the plane of interest are zero, the shear stresses parallel to the cross section plane are zero, and the in-plane stresses are a function of the in-plane coordinates only. Even 
though rock masses exhibit some heterogeneity, anisotropy, and non-elastic behavior (e.g., Leith et $\mathrm{al}^{12}$ ) linear elastic solutions for homogeneous, isotropic, isothermal bodies nonetheless serve as a valuable starting point for understanding the stresses at shallow depths beneath rock slopes (i.e., depths less than the topographic relief). The solutions treated here are based on the small amplitude approximations for periodic topography of Holzhausen ${ }^{2}$ and Haneberg. ${ }^{24}$ These solutions describe a broad range of topographic forms with only two parameters, provide useful insights that are difficult to extract from numerical solutions, can be evaluated quickly on a computer and hence are useful for exploratory analyses, and can be used to develop solutions for two-dimensional topography of arbitrary shape using Fourier series methods. Small modifications to these solutions allow the topographic perturbations of gravitational stresses and tectonic stresses to be better distinguished and compared in new ways that provides useful insights.

This treatment begins by reviewing the ambient stress fields that arise in the absence of topography from just gravity and just a uniform tectonic load. The crux of the manuscript follows: a suite of first-order solutions for near-surface topographic stresses that clearly distinguish between the topographic effects on gravitational body forces and on a uniform regional horizontal stress field for periodic cosinusoidal topography of small amplitude. These solutions are examined separately and then jointly to identify the relative contributions of gravitational stresses and topographic stresses to near-surface stresses over a broad range of conditions. To gauge the accuracy of the first-order solutions, they are compared against numerical solutions that reproduce exact solutions. The results have a variety of practical applications, especially for understanding near-surface fracturing, but the main thrust is to develop general insights into the effect of low amplitude periodic topography on gravitational and tectonic stresses.

\section{Ambient stress fields}

\subsection{Ambient gravitational stress field}

An infinite number of theoretical plane strain stress fields honor the equations of equilibrium

and compatibility ${ }^{25}$ and account for gravitational body forces. One warrants special attention. In 
the absence of any lateral stresses, the ambient stresses due exclusively to the weight of a body of density $\rho$ under a gravitational acceleration $g \operatorname{are}^{25}$ :

$\sigma_{x x}^{g, 0}=0$,

$\sigma_{y y}^{g, 0}=\rho g y$,

$\sigma_{x y}^{g, 0}=\sigma_{y x}^{g, 0}=0$,

where $y$ is positive in the upward direction, $y=0$ coincides with the horizontal ground surface, and the $x$-direction is horizontal (Fig. 1). Normal stresses are considered positive here if tensile (Fig. 1) and negative if compressive. This is consistent with the convention of Holzhausen, ${ }^{2}$ Savage et al, ${ }^{5}$ and Haneberg. ${ }^{24}$ The stress field described by Eqs. (1-3) is referred to here as the ambient gravitational stress field, with superscripts $g$ and 0 .

Two other theoretical reference stress fields associate horizontal stresses with gravitational stresses. One example is that of lithostatic stress, where the horizontal normal stress equals the vertical normal stress ${ }^{26}$ :

$\sigma_{x x}{ }^{\text {lithostatic }}=\rho g y$,

$\sigma_{y y}{ }^{\text {lithostatic }}=\rho g y$,

$\sigma_{x y}^{\text {lithostatic }}=\sigma_{y x}^{\text {lithostatic }}=0$.

Anderson ${ }^{26}$ noted that this is a convenient reference state but "will not often happen in nature." Another theoretically permissible field involves horizontal stresses that vary linearly with depth. Where no horizontal displacement occurs ${ }^{5,27}$ the vertical gradient in the horizontal stress can be expressed in terms of Poisson's ratio $(v)$, yielding the following expressions:

$$
\begin{aligned}
& \sigma_{x x}^{\text {lateral confinement }}=\frac{v}{1-v} \rho g y, \\
& \sigma_{y y}^{\text {lateral confinement }}=\rho g y, \\
& \sigma_{x y}^{\text {lateral confinement }}=\sigma_{y x} \text { lateral confinement }=0 .
\end{aligned}
$$

McGarr and $\mathrm{Gay}^{28}$ noted that this theoretical stress field for laterally confined rock, though permissible, "has rarely been observed."

Of the theoretically possible stress fields, only the ambient stress field of Eqs. (1-3) occurs in the absence of horizontal normal stresses. Since a major aim here is to discriminate between how 
topography perturbs gravitational stresses and horizontal tectonic stresses separately, the ambient stress field of Eqs. (1-3) serves as the reference here for purely gravitational stresses.

\subsection{Ambient tectonic stress field}

The ambient tectonic stress field investigated here is for a constant lateral horizontal normal stress, the simplest non-trivial case:

$\sigma_{x x}{ }^{T, 0}=T$

$\sigma_{y y}^{T, 0}=0$,

$\sigma_{x y}^{T, 0}=\sigma_{y x}{ }^{T, 0}=0$.

This stress field does not include a vertical gradient in the ambient horizontal tectonic stress. The focus of this manuscript, however, is for stresses at shallow depths for topography of small amplitude, and provided that the vertical gradient in the ambient horizontal tectonic stress is small relative to $\rho g$, it can be reasonably neglected.

\section{Two-dimensional solutions for topographic stress perturbations}

The total near-surface stress field is found by summing the total ambient stress field $\left(\sigma_{i j}{ }^{0}\right)$, given by superposing Eqs. (1-3), Eqs. (10-12), and the stress perturbation $\left(\Delta \sigma_{i j}\right)$ resulting from topography:

$\sigma_{i j}{ }^{\text {total }}=\sigma_{i j}{ }^{0}+\Delta \sigma_{i j}$.

The stress perturbations and the total stress fields considered here are for topographic surfaces that are described by a simple periodic function (Fig. 1)

$y_{t}=A \cos (k x)$

where $A$ is the amplitude, $k$ is the wavenumber,

$k=\frac{2 \pi}{L}$,

and $L$ is the wavelength. The angle of the topographic slope $(\beta)$ is

$\beta=\tan ^{-1}\lfloor-A k \sin (k x)\rfloor$. 
The absolute magnitude of the steepest slope is approximately $A k$ (in radians) and occurs at inflection points in the topography. The steepest slopes considered here are $\sim 14^{\circ}$, where $A / L=$ 0.04 and $A k=2 \pi / 25$.

\subsection{Nature of first-order stress approximations}

In the first-order approximations here, the ambient stress state is perturbed such that the normal and shear tractions along the topographic surface are approximately zero. The calculation of the perturbing stresses for low amplitude topography involves approximating the cosine of the slope angle as unity and the sine of the slope angle as the slope angle itself. These approximations become increasingly accurate as the slope approaches zero.

\subsection{Gravitational stresses}

\subsubsection{Stress perturbation}

The first-order stress perturbations of the ambient gravitational stress field can be obtained by retaining just the terms in Eqs. (23)-(25) of Haneberg ${ }^{24}$ with a trigonometric component:

$$
\begin{aligned}
& \Delta \sigma_{x x}{ }^{g}=(-\rho g A)(1+k y)[\exp (k y)][\cos (k x)], \\
& \Delta \sigma_{y y}{ }^{g}=(-\rho g A)(1-k y)[\exp (k y)][\cos (k x)] \text {, }
\end{aligned}
$$

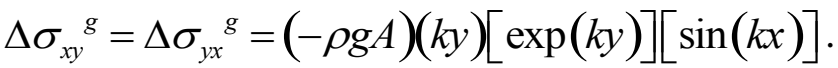

Each of these expressions has the same form, with four terms: A characteristic gravitational stress $(-\rho g A)$, which is the magnitude of the vertical compressive stress beneath a column of rock of height $A$; A second term that varies linearly with elevation $y$; A third term giving an exponential decay of stresses below the mean elevation of the surface; ands a periodic fourth term, consistent with the periodic nature of the topography.

If $A=0$ (i.e., no topographic relief occurs), then the topographic stress perturbations vanish. Far below the topographic surface (i.e., as $y \rightarrow-\infty$ ) the exponential term approaches zero and hence the topographic stress perturbations do as well. The depth to which topography influences the stresses is determined by the wavelength of the topography, not its amplitude, whereas the magnitude of the topographic stress perturbation is proportional to the amplitude. At a distance of half a wavelength below the topographic surface the exponential term is only $\sim 4 \%$, indicating the topographic influence has effectively vanished. Interestingly, Eqs. (17-19) also describe exactly the stresses beneath the surface of an elastic half-plane subject to a periodic 
normal stress of magnitude $-\rho g A \cos (k x) .{ }^{27}$ The first-order topographic stress perturbations of the gravitational stress field thus can be viewed as incorporating an upward continuation (for $y>$ 0 ) of the subsurface stresses $(y<0)$ due to the weight of the topography on the plane $y=0$. As is the case for all plane strain solutions for bodies without cavities where the boundary conditions are defined in terms of stresses, the solutions here (and all subsequent solutions) are independent of the elastic moduli. ${ }^{25}$

\subsubsection{Total stresses}

The total stress field due to gravitational stresses and the perturbing effects of topography are found by superposing the ambient gravitational stress field of Eqs. (1-3) and the stress perturbations of Eqs. (17-19):

$$
\begin{aligned}
& \sigma_{x x}^{g, \text { total }}=(-\rho g A)(1+k y)[\exp (k y)][\cos (k x)], \\
& \sigma_{y y}^{g, \text { total }}=\rho g y-(\rho g A)(1-k y)[\exp (k y)][\cos (k x)], \\
& {\sigma_{x y}{ }^{g, t o t a l}}=\sigma_{y x}{ }^{g, t o t a l}=(-\rho g A)(k y)[\exp (k y)][\sin (k x)] .
\end{aligned}
$$

Equations (17) and (20) match, as do Eqs. (19) and (22), because the corresponding ambient gravitational stress components equal zero. These solutions are consistent with Eqs. (23)-(25) of Haneberg. ${ }^{24}$ Haneberg ${ }^{24}$ incorporated a horizontal normal stress that varied linearly with depth in his solutions for gravitational stresses, and if that contribution is removed the solutions match, aside from a difference in notation. These equations also satisfy the equations of equilibrium and compatibility for two-dimensional problems. ${ }^{25}$

Figure 2 shows the total subsurface in-plane gravitational stress field, based on Eqs. (20-22), in terms of the most tensile in-plane stress ( $\sigma_{1}$, left column), the least tensile in-plane stress ( $\sigma_{2}$, central column), and the trajectories perpendicular to the most tensile in-plane stress (right column) for ratios of $A / L$ of 0.02 (top row), 0.03 (middle row), and 0.04 (bottom row). Note that these figures have no vertical exaggeration and that the stresses are normalized by $\rho g A$. The normalized values at the base of Fig. $2 \mathrm{~h}$ have smaller absolute values than those at the base of Fig. $2 \mathrm{~b}$ because the normalizing factor is larger. Equations for calculating the principal stresses and the orientations of the stress trajectories based on $\sigma_{x x}, \sigma_{y y}$, and $\sigma_{x y}$ may be found in many references (e.g., Timoshenko and Goodier ${ }^{25}$ ). The most tensile stress (Figs. 2a, d, and g) is positive beneath the valleys and is oriented nearly horizontally (Figs. 2c, f, and i). These positive 
horizontal tensile stresses extend a depth of $\sim 0.1 \mathrm{~L}$ to $0.15 \mathrm{~L}$ beneath valley bottoms. The tensile stress reaches its greatest normalized magnitude of $\sim 1-k A$ at valley bottoms. The most tensile stress beneath a ridge is negative (i.e., compressive). The least tensile stress (Figs. 2b, d, and g) is negative (i.e., compressive) everywhere, with stress contours being subparallel to the topographic surface. The vertical stress characteristically becomes monotonically more compressive with depth. Figures $2 \mathrm{c}$, f, and i show that the most compressive in-plane stress accounting for just topography and gravity is nearly vertical, except near the surface where the most compressive stress is either parallel to the surface (beneath the convex part of a ridge) or perpendicular to the topographic surface (beneath the concave part of a valley). The general pattern where the most compressive stress is nearly vertical serves a signature of a gravitationally dominated stress field.

In order to gauge the accuracy of the first-order approximations, they can be compared to numerical solutions. Figure 3 shows boundary element (BEM) solutions where the topographic surface is simulated by a long cosinusoidal traction-free crack; this technique has been shown to the match the exact analytical solutions of Savage et $\mathrm{al}^{5}$ quite well. ${ }^{16,29}$ The region shown in Fig. 3 is along the central wavelength of a cosinusoidal crack 40 wavelengths long that is divided into 2001 elements of nearly equal length. A comparison of Fig. 2 and Fig. 3 shows that the solutions compare favorably. The first-order solutions and BEM solutions are difficult to distinguish for $A / L<0.03$, for which maximum slopes are less than $11^{\circ}$.

\subsubsection{Stresses along the topographic surface due solely to gravity}

Evaluating the total stresses along the topographic surface serves two key purposes. First, the stresses can be compared to the boundary conditions along the surface to gauge the accuracy of the first-order approximations. Since Eqs. (20-22) satisfy the equations of equilibrium and compatibility, if they also satisfy the traction-free boundary conditions along the surface, then

they would yield an exact solution everywhere in the subsurface. Second, the evaluation yields a solution for the stresses parallel to the surface, a quantity that can be measured in the field but that is not determined by the traction-free boundary condition. The stress state at a point along the surface is particularly useful if described in a reference frame with one axis (the $n$-axis) normal the surface and another axis (the $p$-axis) parallel to the surface (Fig. 1). 
The topographic surface is a principal surface. No normal traction $\left(\sigma_{n n}\right)$ or shear traction $\left(-\sigma_{n p}\right)$ acts on it. These tractions, along with the tensile (or compressive) stress acting along the surface $\left(\sigma_{p p}\right)$, can also be calculated from the approximate stresses in Eqs. (20-22) using the stress transformation equations ${ }^{30}$ :

$\sigma_{n n}=a_{n x} a_{n x} \sigma_{x x}+a_{n x} a_{n y} \sigma_{x y}+a_{n y} a_{n x} \sigma_{y x}+a_{n y} a_{n y} \sigma_{y y}$,

$\sigma_{n p}=\sigma_{p n}=a_{n x} a_{p x} \sigma_{x x}+a_{n x} a_{p y} \sigma_{x y}+a_{n y} a_{p x} \sigma_{y x}+a_{n y} a_{p y} \sigma_{y y}$,

and

$\sigma_{p p}=a_{p x} a_{p x} \sigma_{x x}+a_{p x} a_{p y} \sigma_{x y}+a_{p y} a_{p x} \sigma_{y x}+a_{p y} a_{p y} \sigma_{y y}$.

where

$a_{n x}=\sin (\beta)$,

$a_{n y}=\cos (\beta)$,

$\sigma_{p x}=\cos (\beta)$,

and

$a_{p y}=-\sin (\beta)$.

Figure 4 shows that the first-order solutions for the normal and shear stresses (thin broken curves) acting on the topographic surface are near zero along the topographic surface, less than $10 \%$ of $\rho g A$ for $A / L \leq 0.04$. This discrepancy increases substantially as $A / L$ increases. Accordingly, the first-order approximations are not considered here for $A / L>0.04$.

A short first-order approximation for the stress acting parallel to the surface $\left(\sigma_{p p}\right)$ can be found using properties of twice the mean normal in-plane stress, a stress invariant equal to the sum of $\sigma_{x x}$ and $\sigma_{y y} \cdot{ }^{30}$ Accordingly,

$\sigma_{p p}+\sigma_{n n}=\sigma_{x x}+\sigma_{y y}$.

Since along the surface $\sigma_{n n}=0, \sigma_{p p}$ equals twice the mean normal in-plane stress

$\sigma_{p p}=2\left(\frac{\sigma_{x x}+\sigma_{y y}}{2}\right)=\sigma_{x x}+\sigma_{y y}$

Substituting Eqs. (20) and (21) for $\sigma_{x x}$ and $\sigma_{y y}$, respectively, yields the first-order approximation for $\sigma_{p p}$ due just to gravity: 
${\sigma_{p p}}^{\text {g,total }}=\sigma_{x x}^{\text {g,total }}+\sigma_{y y}^{\text {g,total }}=\rho g A \cos (k x)-2 \rho g A[\exp (k y) \cos (k x)]$.

Substituting Eq. (14) for $y_{t}$ into Eq. (32) yields an even simpler expression:

$\sigma_{p p}^{g, t o t a l}=\rho g y_{t}\left[1-2 \exp \left(k y_{t}\right)\right]$.

For topography with small slopes $(A / L \leq 0.04)$, the exponential term is greater than 0.5 , so the term in square brackets in Eq. (33) is negative everywhere. This indicates that along the ridges $\left(y_{t}>0\right)$ the contribution to $\sigma_{p p}$ due just to gravity will be compressive, whereas along valleys $\left(y_{t}<0\right)$ this contribution will be tensile (Fig. 4).

A second way to gauge the accuracy of these first-order approximations is to compare them to numerical solutions. The thick curves in Figure 4 show boundary element (BEM) solutions. ${ }^{31}$ The BEM results match the no-traction boundary conditions well and therefore are considered to be more accurate than the first-order approximations.

Both the first-order solutions and the BEM solutions predict the stress parallel to the surface $\left(\sigma_{p p}\right)$ is positive (tensile) at valley bottoms and compressive (negative) at ridge crests (Fig. 4). The first-order solutions, however, overestimate the compressive stresses at the ridge crest by as much as a factor or three and overestimate the tensile stresses at the valley bottoms compared to the BEM solutions. The overestimate of the most compressive stress at the ridge crest by the first-order approximations can also be seen by comparing Fig. $2 \mathrm{~h}$ and Fig. $3 \mathrm{~h}$. The BEM solutions indicate that the stress parallel to the surface due to gravity has maxima and minima of approximately $\pm \rho g A / 2$, with these values being relatively insensitive to the $A / L$ ratio. Somewhere between the ridge crests and valley bottoms, the surface parallel-stress due to gravity must equal zero. The first-order solutions (thin solid curves) indicate these "null points" along the topographic surface occur where $y=0$ (e.g., $x / L= \pm 0.25$ ), at inflection points where the elevation equals the mean topographic elevation. In contrast, the BEM solutions (Fig. 4, thick solid curves) indicate that these null points occur in valleys (e.g., at $x / L \approx \pm 0.35$ ).

\subsection{Tectonic stresses}

\subsubsection{Stress perturbation}

The first-order perturbing stress field for a uniform horizontal tectonic stress $T$ is $^{2}$

$$
\Delta \sigma_{x x}{ }^{T}=(T)[-k A(k y+2)][\exp (k y)][\cos (k x)],
$$


$\Delta \sigma_{y y}{ }^{T}=(T)[k A(k y)][\exp (k y)][\cos (k x)]$,

$\left.\left.\Delta \sigma_{x y}^{T}=(T)\lfloor-k A(k y+1)\rfloor \exp (k y)\right\rfloor \sin (k x)\right\rfloor$.

These equations also honor the requirements for equilibrium and compatibility. A comparison of Eqs. (34-36) with Eqs. (17-19) shows that the first-order stress perturbations for tectonic stresses have forms similar to the first-order gravitational stress perturbations, with four terms: a characteristic stress, which here is the horizontal tectonic stress $(T)$; a second term that varies linearly with elevation $y$; a third term that yields an exponential decay of stresses below the mean elevation of the surface; and a periodic fourth term. The similar forms of the gravitational and tectonic stress perturbations aid in comparing and combining them, as will be done subsequently.

\subsubsection{Total stresses}

The ambient horizontal tectonic stress $T$ needs to be accounted for to obtain the total stress field. The total horizontal stress is

$\left.\sigma_{x x}^{T, \text { total }}=T-T\lfloor k A(k y+2)\rfloor\lfloor\exp (k y)\rfloor \cos (k x)\right\rfloor$.

For the remaining components, the ambient values of $\sigma_{y y}$ and $\sigma_{x y}$ are zero, so the perturbing stress equals the total stress, hence

$\sigma_{y y}{ }^{T, \text { total }}=T[k A(k y)][\exp (k y)][\cos (k x)]$,
$\sigma_{x y}^{T, \text { total }}=\sigma_{y x}{ }^{T, \text { total }}=T[-k A(k y+1)][\exp (k y)][\sin (k x)]$.

Figure 5 shows the total subsurface in-plane tectonic stress field in terms of the most tensile in-plane stress ( $\sigma_{1}$, left column), the least tensile in-plane stress $\left(\sigma_{2}\right.$, central column), and the trajectories perpendicular to the most tensile in-plane stress (right column) for ratios of $A / L$ of 0.02 (top row), 0.03 (middle row), and 0.04 (bottom row). The stresses in Fig. 5 are normalized by $-T$. The reader should keep in mind that the stresses in Fig. 5 occur in the absence of gravitational stresses.

The most tensile near-surface stress due exclusively to tectonic loads (Figs. 5a, d, and g) generally is positive beneath the ridges, negative beneath the valleys, and nearly vertical (Figs. $5 \mathrm{c}, \mathrm{f}$, and i). The vertical stress reaches its greatest tensile magnitude of $(2 \pi T / e)(A / L)$ at an elevation of $y=-1 / k=-L / 2 \pi$ beneath a ridge crest (Fig. 5, left column). The vertical stress 
reaches its greatest compressive value of $(-2 \pi T / e)(A / L)$ at an elevation of $y=-1 / k=-L / 2 \pi$ beneath a valley bottom. Interestingly, the elevations of these maxima and minima are independent of the amplitude of the topography in the small amplitude approximations. These results imply that very strong regional compressive stresses could cause fractures to open beneath ridges, with the fractures being subparallel to the topographic surface.

The least tensile near-surface stress due exclusively to tectonic loads is negative (i.e., compressive) everywhere beneath topography of small amplitude (Figs. 5b, d, and g). At a given elevation, the stresses are more compressive beneath a valley than a ridge. This can be understood in terms of a horizontal force balance. The total horizontal force across every vertical plane through the body must be the same for equilibrium. Since the height of a vertical section is the least beneath a valley, the average horizontal stress must be more compressive along a section beneath a valley than beneath a ridge. In the near-surface plots of Fig. 5 the absolute values of the least tensile (most compressive) stresses are far greater than the absolute values of the most tensile stress almost everywhere for a uniform tectonic stress in the absence of gravitational stresses. The least tensile stress effectively matches the horizontal tectonic stress at an elevation of $-0.3 L$, indicating that the topographic perturbations of the horizontal tectonic stress have effectively vanished there.

Figures $5 \mathrm{c}, \mathrm{f}$, and $\mathrm{i}$ indicate that the most compressive stress is subhorizontal everywhere for a uniform horizontal tectonic stress in the absence of gravity. The general pattern where the most compressive stress is nearly horizontal serves a signature of a stress field dominated by compressive horizontal tectonic stresses beneath topography of small amplitude.

To help gauge the accuracy of the first-order approximations for topographically perturbed tectonic stresses, they can be compared to numerical solutions, as done previously for the perturbed gravitational stresses. Figure 6 shows BEM solutions, calculated in the manner of Fig. 3. An inspection of Fig. 5 and Fig. 6 shows that the solutions generally compare favorably below the surface for $A / L \leq 0.03$ (top two rows), for which maximum slopes are less than $11^{\circ}$. The stress trajectory patterns (right columns) are quite similar. Perhaps the most prominent discrepancies between the first-order approximations and BEM solutions occur at the ridge crest. The first-order approximations indicate the most compressive stress there (Figs. 5b, e, h) is somewhat less compressive than the BEM solutions (Figs. 6b, e, h). Also, the first-order approximations indicate the most tensile stress at the ridge crest is negative (Figs. 5a, d, g), 
whereas the BEM solutions show the most tensile stress there is positive (Figs. 6a, d, g). The reason for this is discussed in the next section.

\subsubsection{Stress along the topographic surface due to solely to the horizontal tectonic stress}

The total stresses along the topographic surface arising from horizontal tectonic loads alone are calculated in the same manner used to evaluate effects of gravity. The stresses acting on the surface $\left(\sigma_{n n}, \sigma_{n p}\right)$ resulting from just a uniform horizontal tectonic stress are obtained by inserting Eqs. (37-39) into Eqs. (23-25). These stresses would have a value of zero if they matched the boundary conditions perfectly. Figure 7 shows that the calculated normal stresses (dashed curves) are compressive, being a few percent of $T$ for $A / L \leq 0.02$, but locally reaching $\sim 20 \%$ of $T$ for $A / L=0.04$. The calculated shear stresses (dotted curves) have a mean value of zero but are locally about as high as $0.25|T|$ for $A / L=0.02,0.36|T|$ for $A / L=0.03$, and $0.46|T|$ for $A / L=0.04$. The first-order perturbation solutions for a uniform regional horizontal stress have substantially greater errors in terms of the corresponding characteristic stress than the solutions for topographically perturbed gravitational stresses. No ratios of $A / L$ greater than 0.04 will be considered here as a consequence of these errors.

First-order expressions for the surface-parallel stress $\left(\sigma_{p p}\right)$ are obtained by inserting Eqs. (37) and (38) into Eq. (31):

$\sigma_{p p}{ }^{T, t o t a l}=T\left[1-2 k A \exp \left(k y_{t}\right) \cos (k x)\right]$.

or

$\sigma_{p p}{ }^{T, t o t a l}=T\left[1-2 k y_{t} \exp \left(k y_{t}\right)\right]$.

For topography with small slopes $(A / L \leq 0.04)$, even though the exponential term is greater than 0.5, the $k y_{t}$ term in Eq. (41) generally is small relative to unity, so the sign of the surface-parallel stress is of the same sign as $T$ all along a topographic surface of small amplitude.

Figure 7 compares the first-order solutions (thin curves) and the BEM solutions (thick curves) for stress components at the small-amplitude topographic surface for a uniform horizontal tectonic stress. Both sets of solutions show key qualitative similarities. The surface-

parallel stress $\left(\sigma_{p p}\right)$ is compressive everywhere along the small amplitude surface. This stress is more compressive than the regional horizontal stress at the valley bottoms, and less compressive 
at the ridge crests. The compression at the ridge crest also diminishes as the $A / L$ ratio increases. Both solutions predict that the surface-parallel stress will match the ambient lateral tectonic stress at or very near points on the surface at the mean elevation of $y=0$; in Fig. 7 these points are at $x / L= \pm 0.25$. The BEM solutions for the stress components acting on the topographic surface (thick broken lines) essentially equal zero, matching the boundary conditions, whereas the first-order solutions (thin broken curves) show discernible departures from the boundary conditions even for values of $A / L$ of 0.02 . The BEM solutions thus honor the no-traction boundary condition better than the first-order approximations, as was the case for the solutions for gravitational stresses. The negative (compressive) values of $\sigma_{n n}$ in the first- order approximations explain a noteworthy discrepancy between Figs. 5 and 6 . In the first-order solutions, the most tensile stress immediately below the ridge crest is compressive (Fig. 5, left column), whereas the BEM solutions show a tension (Fig. 6, left column). Both solutions show that the stress parallel to the surface is compressive at the ridge crest, which is convex, so the stress normal to the surface immediately below the ridge crest must be tensile $\mathrm{e}^{22}$ as shown in Fig. 6. The region immediately below the ridge crest in Fig. 5 where $\sigma_{1}$ is shown as negative (compressive) is an incorrect artifact of the first-order approximation. The first-order approximations thus underestimate the most tensile stress immediately below ridges.

\section{Solutions for gravity combined with a uniform horizontal tectonic stress}

The topographically perturbed gravitational field (Figs. 2 and 3) and the topographically perturbed tectonic stress field (Figs. 5 and 6) represent distinct end members of a spectrum in terms of both the magnitude of and orientation of the principal stresses. Conditions in nature would likely lie between these end members. The focus now is how weak the horizontal tectonic stress needs to be for gravitational stresses to dominate the near-surface stress field beneath periodic topography of small amplitude, and how large the horizontal tectonic stress needs to be for tectonic stresses to dominate. The treatment here utilizes the small-amplitude approximations, which have been shown to be sufficiently accurate for $A / L \leq 0.04$.

The contributions of the first-order topographic perturbations for gravitational and tectonic stresses solutions can be combined and compared readily owing to their forms. Recasting the tectonic stress $T$ in terms of the characteristic vertical stress due to gravity is the critical step:

$T=-n \rho g A$. 
If, for example, $n=+10$, then the ambient horizontal tectonic stress is compressive and ten times the magnitude of the characteristic vertical stress due to gravity. Substituting Eq. (42) into Eqs. (37-39) and then summing with Eqs. (20-22) yields the total first-order topographically perturbed stress field for gravitational and tectonic stress combined. Substituting Eq. (42) into Eq. (40) and then summing with Eq. (32) yields the total first-order approximation for the surface-parallel stress. The equations for the total solutions are not reproduced here because they do not yield sufficient additional insight into the total stress fields, but graphical solutions do.

Figure 8 shows normalized stress fields for $n=0.1$, with a normalization factor of $-T=$ $n \rho g A$. The plots of Fig. 8 are qualitatively similar to those for a gravitational body force alone (Fig. 2): the shapes of the corresponding stress contours are similar, as are the orientations of the trajectories normal to the most tensile stress. The values of the stresses also would be similar if they had the same normalization factors: the normalization factor for Fig. 2 is ten times that for Fig. 8, and the normalized values for Fig. 2 are about $10 \%$ of those in Fig. 8. Additionally, the most compressive stress is essentially vertical except near the ridge crest: this is a signature of a stress field dominated by effects of gravity. For topography of small amplitude, if $n<0.1$ then the near-surface stress field is essentially dominated by gravitational stresses.

Figure 9 shows normalized stress fields for $n=1$, with a normalization factor of $-T=\rho g A$. The plots of Fig. 9 generally are qualitatively similar to those for cases where gravitational body force act by themselves (Fig. 2) but exhibit some quantitative differences. The contours of the most tensile stress (left column) in Fig. 9 have shapes crudely similar to their counterparts in Fig. 2 , but have normalized values that are less by about -1 ; this reflects the effect of the regional horizontal compressive stress in Fig. 9. The values of the most tensile stress for $n=1$ thus reflect a combination of gravitational effects and tectonic effects. The plots of the least tensile stress (central column) and the stress trajectories (right column) in Fig. 9 are similar qualitatively and quantitatively to the corresponding plots in Fig. 2, although some discrepancies appear for $A / L=0.04$. The character of the near-surface stress field for $n=1$ thus is more clearly influenced by gravitational stresses than by tectonic stresses, but effects of both end members are discernible.

Figure 10 shows normalized stress fields for $n=10$, where the normalization factor is $-T=$ $10 \rho g A$. Note that along a given row $\left(A / L\right.$ ratio) in Fig. 10, the normalized contour plots of $\sigma_{1}$ (left column), the normalized contour plots of $\sigma_{2}$ (central column), and the stress trajectory plots 
(right column) are divided into upper and lower portions of distinctly different character, with the boundary coinciding approximately with the base of the U-shaped contour with a value of -1 in the normalized contour plot of $\sigma_{2}$. This boundary is at $y / L \approx-0.2,-0.3$, and -0.4 for the first, second, and third rows, respectively. The upper portions of the stress trajectory plots in Fig. 10 show that the most compressive stress trajectories essentially parallel the topographic surface, as is the case in the upper parts of the plots where tectonic stresses act in the absence of gravity (Fig. 5). In addition, the upper portions of the normalized contour plots of $\sigma_{2}$ in Fig. 10 resemble those where tectonic stresses act in the absence of gravity (Fig. 5). In contrast, in the lower portions of Fig. 10 the most compressive stress trajectories are essentially vertical, the contours of the most compressive stress are parallel to the surface, and the contours of the most tensile stress become vertical, as is the case in the lower parts of the plots where gravity acts in the absence of tectonic stresses (Fig. 2). In sum, for $n=10$, the character of the stress field shows a mix of and gravitational influences, with tectonic influences dominating at very shallow depths $(y / L>-0.2)$, and gravitational influences dominating at greater depths. Additionally, a comparison of the rows in Fig. 10 shows that as the horizontal tectonic compressive stress increases (i.e., as $A$ increases), its signature becomes more pronounced at increasingly greater depths.

Figure 11 shows normalized stress fields for $n=100$. The normalization factor is $-T=$ $100 \rho g A$. The plots of Fig. 11 are qualitatively similar in some key ways to those where tectonic stresses act by themselves (Figs. 5, 6). First and foremost, in each case the trajectories of the most compressive stress are subparallel to the surface. The shapes and values of the contours of the normalized least tensile stress $\left(\sigma_{2} /-T\right)$ are similar. The absolute values of the magnitudes of the normalized most tensile stress $\left(\sigma_{1} /-T\right)$ are much less than those of the normalized least tensile stress $\left(\sigma_{2} /-T\right)$. These comparisons show that for topography of small amplitude, if $n>$ 100 then the near-surface stress field is dominated by the effects of uniform tectonic stresses for $y / L>-0.4$.

The differences among Figs. 5, 6, and 11 are most apparent in the distributions of the normalized most tensile stress $\left(\sigma_{1} /-T\right)$. For example, Fig. 11 shows a monotonic decrease in $\sigma_{1} /-T$ with depth beneath the valleys, whereas Figs. 5 and 6 show localized minima beneath the valleys at $y / L \approx-0.15$; this difference reflects the presence of gravitational stresses in Fig. 11 . 
Also, Fig. 5 and Fig. 11 show the most tensile stress immediately beneath the ridge crests is negative (compressive), whereas Fig. 6 shows a tension; the ridge crest compressive stress concentrations in Figs. 5 and 11 reflect inaccuracies in the first-order approximations (see section 3.3.3). Boundary element solutions (not shown here) honoring the loading of Fig. 11 in fact show a vertical tension immediately below the ridge crest for all three $A / L$ ratios.

\section{Discussion}

The main thrust of this contribution is to identify and compare the effects of periodic topography of small amplitude on near-surface stress fields arising from uniform lateral tectonic loads and from gravity. The discussion first treats six germane points: (1) the effect of material properties on topographic stress perturbations; (2) the meaning of the first-order perturbation solution for gravitational stresses; (3) conditions that determine whether the effects of gravity or tectonic stresses dominate the stress field at shallow depths; (4) how the results compare with solutions for bell-shaped topography ${ }^{5,6}$; (5) implications for near-surface fracturing; and (6) implications for stress measurements.

For plane strain and plane stress solutions with traction boundary conditions, a vertical gravitational body force ( $\rho g)$, and a horizontal body force that does not vary with horizontal position, the stress field generally is independent of the elastic moduli. ${ }^{25}$ This is the situation considered here. The dependence of the gravitationally induced stress field on Poisson's ratio described by Savage et $\mathrm{al}^{5}$ arises because they define the ambient gravitational stress field in terms of Poisson's ratio in the manner of Eqs. (7-9) for the special case of a laterally confined body. For a broad range of two-dimensional problems, including those considered here, the topographic stress perturbations in a uniform, isotropic, isothermal material (a) depend only on the horizontal tectonic stresses, gravitational acceleration, the density of the rock and the geometry of the body; and (b) are entirely independent of the elastic moduli unless the horizontal tectonic stresses are defined in terms of the elastic moduli.

The first-order approximate solution for the perturbation of gravitational stresses by periodic topography (see Eqs. 17-19) matches the exact analytical solution for the stresses beneath the surface of an elastic half-plane subject to a periodic normal traction of magnitude $-\rho g A \cos (k x)$ and no shear traction. ${ }^{27}$ Stated alternatively, the first-order approximate solution to the exact topographic problem is in fact the exact solution to a related approximate problem. To help 
understand why, first note that the solution of Eqs. (17-19) when combined with the ambient gravitational stress field yields normal and shear tractions along a topographic surface of small amplitude ( $\sigma_{n n}$ and $\sigma_{n p}$, respectively, in Fig. 4) that are nearly equal to zero; the traction-free boundary condition along the topographic surface thus is nearly honored. Second, the horizontal shear stress is exactly zero along the surface of the half-plane in the problem for the periodic normal loading, and the shear-traction boundary condition for that problem is exactly honored by Eqs. (17-19). Third, the periodic normal traction of magnitude $-\rho g A \cos (k x)$ effectively matches the load imposed by columns of rock that extend from the mean elevation to the cosinusoidal topographic surface. The exact solution for the periodic loading problem thus nearly honors the boundary conditions of the topographic problem. The first-order approximation breaks down for topography of large amplitude not because of a violation of the equations of compatibility and equilibrium or because of numerical inaccuracies, but rather because the topography departs substantially from that of a plane, causing the honoring of the traction-free boundary condition at the topographic surface to decay.

The magnitude and orientation of the principal stresses in the shallow subsurface provide the basis for discriminating between stress fields dominated by gravity as opposed to those dominated by the ambient horizontal tectonic compressive stress. For topography of small amplitude where gravity dominates (Fig. 3), the most compressive stress is nearly vertical, the contours of the most compressive stress nearly parallel the surface, the surface-parallel stress is most compressive at ridge crests and most tensile at valley bottoms, and the stresses normal to the surface are compressive beneath ridges at shallow depths. In contrast, where the horizontal tectonic stress dominates (Fig. 5), the most compressive stress is nearly horizontal, the contours of the least compressive stress generally are distinctly oblique to the surface, the surface-parallel stress is most compressive at valley bottoms and least compressive at ridge crests, and the stresses normal to the surface can be tensile beneath ridges. A review of Figs. 2 and 9 shows that if the magnitude of the uniform horizontal tectonic compression is less than $\sim 0.1 \rho g A$, then the total stress field at very shallow depths $(y / L>-0.2)$ is dominated by the effects of gravity. A review of Figs. 5 and 11, however, shows that if a uniform horizontal tectonic compression is greater than $\sim 10 \rho g A$, then the total stress field at very shallow depths is dominated by the effects of the horizontal tectonic stress; in particular, the orientations of the principal stresses and 
the magnitude of the most compressive stress will be nearly identical to those due just to tectonic loads.

A few of the results from the small amplitude solutions for periodic topography can be readily compared to the analytical solutions for bell-shaped topography ${ }^{5,6}$ (Fig. 1, inset). Two of the main points developed here are (1) that a tectonic compressive stress, if large enough, can induce a tension perpendicular to the surface near the crests of ridges; and (2) that gravitational stresses can induce a tension parallel to the surface beneath valleys. The topographic relief $(b)$ and a positive width parameter $(a)$ define the bell-shaped topography. For a ridge $b>0$; for a valley bottom $b<0$ and $|b|<a$. For gravitational stresses alone, the surface-parallel stress at a ridge crest or valley bottom reduces to $\sigma_{p p}{ }^{g}(y=b)=-\rho g b\lfloor a /(a+b)\rfloor$. This shows that the topographically perturbed surface-parallel stress due to gravity alone will be negative (compressive) at a bell-shaped ridge crest where $b$ is positive, and positive (tensile) at a bellshaped valley bottom where $b$ is negative, consistent with the small amplitude solutions. Alternatively, for a uniform horizontal tectonic stress $T$ in the absence of gravity, the surfaceparallel stress at a bell-shaped ridge crest or valley bottom is $\sigma_{p p}{ }^{T}(y=b)=T\{(2-b / a) /[(2+b / a)(1+b / a)]\}^{6} \quad$ For a bell-shaped ridge or valley of small relief, where $|b|<a$, the surface-parallel stress is compressive at both a ridge crest and a valley bottom. This means the stress normal to the surface will be tensile immediately beneath the convex ridge crest and compressive immediately beneath the concave valley bottom. ${ }^{22}$ The endmember solutions for the stresses beneath periodic topography of small amplitude and bellshaped topography of small relief thus have some key parallels.

The balance between gravitational and tectonic stress contributions has ramifications for near-surface fracturing beneath topography of small amplitude. Two different fracture criteria are considered here, opening mode fracturing and shear fracturing, and only dry isothermal rock is treated.

Fractures can open in dry rock where tensile stresses arise. ${ }^{32}$ Where gravitational stresses act by themselves (Fig. 3), and no pre-existing flaws exist that might cause localized tensile stress concentrations, only beneath valleys do tensile stresses arise within a distance of $0.1 \mathrm{~L}$ of the topographic surface. The most tensile stresses beneath valleys would be nearly horizontal, and thus could cause nearly vertical fractures to open there. Gravitational stresses by themselves do 
not yield a subsurface tension perpendicular to the surface anywhere beneath slopes of small amplitude and hence are unable to cause sheeting joints to open on gentle slopes. This does not mean, however, that gravitational stresses are unable to cause sheeting joints to open on steep slopes. ${ }^{33}$ In contrast, tectonic stresses can be sufficient to overcome the effects of gravity to result in a tension perpendicular to the slope beneath the flanks and crests ridges of small amplitude and cause sheeting joints to open there (left columns of Figs. 6, 10, and 11).

The susceptibility for shear fracture can be gauged using the shear failure potential $\Phi$, the absolute value of the ratio of the maximum shear stress to the mean normal stress. ${ }^{34}$ In terms of the principal stresses, $\Phi=\left|\left(\sigma_{1}-\sigma_{2}\right) /\left(\sigma_{1}+\sigma_{2}\right)\right|$. Increasing values of $\Phi$ indicate increasing susceptibility to shear fracture. Figure 12 shows $\Phi$ for $A / L=0.03$ for four different values of uniform regional compressive stresses. For a small regional compression $(T=-0.1 \rho g)$ valley bottoms and a deep region beneath ridges are most susceptible to shear fracture, and a lenticular region beneath a ridge crest is least susceptible (Fig. 12a). As the regional horizontal stress becomes more compressive, the regions of highest and lowest $\Phi$ values change their size, shape, and location. For $T=-1 \rho g A$ (Fig. 12b) the region of highest $\Phi$ values occurs along a thin region along the ground surface, with the lowest $\Phi$ values in lenticular regions beneath both the ridges and valleys. For $T=-10 \rho g A$ (Fig. 12c) the region of highest $\Phi$ values occurs along a thin region along the ridge flanks, with the lowest $\Phi$ values at great depth. For the most compressive regional stress considered here, $T=-100 \rho g A$ (Fig. 12d), the region of highest $\Phi$ values mirrors the topography, being deepest beneath ridges and shallowest beneath valleys, with the region of lowest $\Phi$ values at great depth. These results are consistent with the three-dimensional results of St. Clair et al. ${ }^{14}$

The theoretical solutions have two clear implications for stress measurements beneath topography of low amplitude. First, the topographic stress perturbations die out by a depth of about half the topographic wavelength. Horizontal stress measurements at a greater depth could yield values close to the regional horizontal stresses provided that the measurements are not made near features such as faults or underground excavations. Second, at certain places near the mean elevation of periodic topography of small amplitude, shallow measurements of the surfaceparallel stress theoretically could yield values near the regional horizontal tectonic stresses. This theoretical prediction might apply to some natural settings, but probably not all because of the 
many wavelengths and steep slopes present in many places. Nonetheless, modeling of topographic stress perturbations might be helpful in the planning stages of stress measurements to reveal the degree of topographic perturbations of the regional stresses.

\section{Conclusions}

Theoretical first-order approximate elastic solutions provide insight into the variety of stress fields that can be expected beneath periodic two-dimensional topography of amplitude $A$ and wavelength $L$ subject to gravity and a uniform horizontal tectonic stress. These solutions for uniform isotropic rock compare favorably with boundary element solutions provided $A / L<0.04$. They are independent of elastic moduli unless the tectonic stress is defined in terms of the elastic moduli. Topographic effects decay to negligible levels at depths below the surface of $\sim L / 2$. Stress measurements at depths greater than $L / 2$ below the mean elevation, as well as measurements at shallow depths near the mean elevation, theoretically could yield good estimates of the tectonic (regional) horizontal stress with little or no influence by gravitational stresses. Inaccuracies in the approximate solutions are most apparent are at ridge crests.

The topographic perturbations of the stress fields due to gravity and a uniform horizontal tectonic compression have similar forms but different effects. Gravity, by itself, causes horizontal compression at the crest of a ridge, horizontal tension at the bottom of a valley, and trajectories perpendicular to the most tensile stress to generally be vertical. Gravitational effects by themselves could cause vertical fractures to open beneath valleys but would not cause surface-parallel sheeting joints to open beneath topography of small amplitude. A uniform horizontal tectonic compression $T$, by itself, causes vertical tension in the subsurface below the crest of a ridge, and horizontal tension at the bottom of a valley. Tectonic effects by themselves could cause surface-parallel sheeting joints to open beneath ridges of small amplitude.

The balance between gravitational and tectonic stresses dictates the nature of the near-surface stress field. If $T$ is an order of magnitude less compressive than $\rho g A$, where $\rho$ is rock density, $g$ is gravitational acceleration, and $A$ is the topographic amplitude, then effects of gravity dominate effects of the regional compression near the topographic surface. Conversely, if $T$ is an order of magnitude more compressive than $\rho g A$, then effects of regional compression dominate the effects of gravity near the surface, and tensile stresses can develop normal to the surface beneath 
convex ridges. The latter conditions, together with the evolution of topography, promote the widespread development of sheeting joints.

Further research is necessary to better understand how topography perturbs the stress field in the shallow subsurface and affects fracturing there. Outstanding topics include the general effects of high-amplitude topography $(A / L>0.04)$, three-dimensional topography, and a vertical gradient in the horizontal tectonic stresses. Effects of water on subsurface fracturing induced by topographic stress perturbations also warrants investigation.

\section{Acknowledgements}

Support from the National Science Foundation to Martel (grant EAR-05-38334), the Yosemite Conservancy (Agreement CA\#H80800090008), and the Army Research Office (Prime award \# W911NF-14-1-0037) is gratefully acknowledged. The review of the manuscript by William Haneberg is gratefully acknowledged. This is SOEST (School of Ocean and Earth Science and Technology, University of Hawaii) contribution 9641. 


\section{References}

1. Jeffreys H. The Earth, Its Origin, History and Physical Constitution. 1st ed. Cambridge: University Press; 1924.

2. Holzhausen GR. Sheet structure in rock and some related problems in rock mechanics. 1977;(December). http://sul-derivatives.stanford.edu/derivative?CSNID=00016262\&mediaType=application/pdf.

3. McTigue DF, Mei CC. Gravity-induced stresses near topography of small slope. J Geophys Res. 1981;86(B10):9268-9278. doi:10.1029/JB086iB10p09268.

4. McTigue DF, Mei CC. Gravity-induced stresses near axisymmetric topography of small slope. Int J Numer Anal Methods Geomech. 1987;11(3):257-268. doi:10.1002/nag.1610110304.

5. Savage WZ, Swolfs HS, Powers PS. Gravitational stresses in long symmetric ridges and valleys. Int J Rock Mech Geomech Abstr. 1985;22(5):291-302. doi:10.1016/0148-9062(85)92061-3.

6. Savage WZ, Swolfs HS. Tectonic and gravitational stress in long symmetric ridges and valleys. J Geophys Res. 1986;91(B3):367-3685. doi:10.1029/JB091iB03p03677.

7. Liu, L., Zoback MD. The effect of topography on the state of stress in the crust: application to the site of the Cajon Pass Scientific Drilling Project. J Geophys Res. 1992;97(B4):5095-5108. doi:10.1029/91JB01355.

8. Pan E, Amadei B, Savage W. Gravitational and tectonic stresses in anisotropic rock with irregular topography. Int J Rock Mech Min Sci Geomech Abstr. 1995;32(3):201-214. http://www.sciencedirect.com/science/article/pii/0148906294000466.

9. Miller DJ, Dunne T. Topographic perturbations of regional stresses and consequent bedrock fracturing. $J$ Geophys Res. 1996;101(B11):25,523-525,536. doi:10.1029/96JB0253.

10. Molnar P. Interactions among topographically induced elastic stress, static fatigue, and valley incision. $J$ Geophys Res. 2004;109(F2):1-9. doi:10.1029/2003JF000097.

11. Mitchell KJ. Factors contributing to the formation of sheeting joints: a study of sheeting joints on a dome in Yosemite National Park. 2010. https://www.soest.hawaii.edu/GG/resources/theses/MS_2010_Mitchell.pdf.

12. Leith K, Moore JR, Amann F, Loew S. In situ stress control on microcrack generation and macroscopic extensional fracture in exhuming bedrock. J Geophys Res Solid Earth. 2014;119(1):594-615. doi:10.1002/2012JB009801.

13. Slim M, Perron JT, Martel SJ, Singha K. Topographic stress and rock fracture: a two-dimensional numerical model for arbitrary topography and preliminary comparison with borehole observations. Earth Surf Process Landforms. 2015;40(4):512-529. doi:10.1002/esp.3646.

14. St. Clair J, Moon S, Holbrook WS, et al. Geophysical imaging reveals topographic stress control of bedrock weathering. Science (80- ). 2015;350(6260):534-539. doi:10.1126/science.aab2210.

15. Shea-Albin VR, Dolinar DR, Peters DC. Calculation of Vertical Stress Exerted by Topographic Features. Washington, D.C.; 1992. http://www.cdc.gov/niosh/mining/userfiles/works/pdfs/ri9409.pdf.

16. Martel SJ, Muller JR. A two-dimensional boundary element method for calculating elastic gravitational stresses in slopes. Pure Appl Geophys. 2000;157(6-8):989-1007. doi:10.1007/s000240050014.

17. Fiske RS, Jackson ED. Orientation and growth of Hawaiian volcanic rifts: the effect of regional structure and gravitational stresses. Proc R Soc A Math Phys Eng Sci. 1972;329(1578):299-326. doi:10.1098/rspa.1972.0115.

18. Dieterich JH. Growth and persistence of Hawaiian volcanic rift zones. J Geophys Res. 1988;93(B5):42584270. doi:10.1029/JB093iB05p04258.

19. McGovern PJ, Solomon SC. State of stress, faulting and eruption characteristics of large volcanoes on Mars. J Geophys Res. 1993;98(93):13,609-613,617.

20. Morin RH, Savage WZ. Topographic stress perturbations in southern Davis Mountains, west Texas 2. Hydrogeologic implications. J Geophys Res. 2002;107(B12):1-10. doi:10.1029/2001JB000488.

21. Savage WZ, Morin RH. Topographic stress perturbations in southern Davis Mountains, west Texas 2. Hydrogeologic implications. J Geophys Res. 2002;107(B12):1-10. doi:10.1029/2001JB000488.

22. Martel SJ. Effect of topographic curvature on near-surface stresses and application to sheeting joints. Geophys Res Lett. 2006;33(1):1-5. doi:10.1029/2005GL024710.

23. Martel SJ. Mechanics of curved surfaces, with application to surface-parallel cracks. Geophys Res Lett. 2011;38(20):1-6. doi:10.1029/2011GL049354.

24. Haneberg WC. Effects of valley incision on the subsurface state of stress - theory and application to the Rio Grande Valley near Albuquerque, New Mexico. Environ Eng Geosci. 1999;5(1):117-131. doi:10.2113/gseegeosci.V.1.117. 
25. Timoshenko SP, Goodier JN. Theory of Elasticity. 3rd ed. New York: McGraw-Hill; 1970.

26. Anderson EM. The Dynamics of Faulting and Dyke Formation with Applications to Britain. 2 nd ed. Edinburgh: Oliver and Boyd; 1951.

27. Jaeger JC, Cook NGW, Zimmerman RW. Fundamentals of Rock Mechanics. 4th ed. Oxford: WileyBlackwell; 2007.

28. McGarr A, Gay NC. State of stress in the Earth's crust. Annu Rev Earth Planet Sci. 1978;6:405-436. doi:10.1146/annurev.ea.06.050178.002201.

29. Martel SJ. Modeling elastic stresses in long ridges with the displacement discontinuity method. Pure Appl Geophys. 2000;157(6-8):1039-1057. doi:10.1007/s000240050016.

30. Chou PC, Pagano NJ. Elasticity - Tensor, Dyadic, and Engineering Approaches. Mineola, New York: Dover; 1992.

31. Crouch SL, Starfield AM. Boundary Element Methods in Solid Mechanics. London: George Allen \& Unwin; 1983.

32. Pollard DD, Segall P. Theoretical displacements and stress near fractures in rock: with applications to faults, joints, veins, dikes, and solution surfaces. In: Atkinson BK, ed. Fracture Mechanics of Rock. London: Academic Press; 1987:227-349. doi:10.1016/B978-0-12-066266-1.50013-2.

33. Stock GM, Martel SJ, Collins BD, Harp EL. Progressive failure of sheeted rock slopes: The 2009-2010 Rhombus Wall rock falls in Yosemite Valley, California, USA. Earth Surf Process Landforms. 2012;37(5):546-561. doi:10.1002/esp.3192.

34. Iverson RM, Reid ME. Gravity-driven groundwater flow and slope failure potential: 1. Elastic effectivestress model. Water Resour Res. 1992;28(3):925-938. doi:10.1029/91WR02694. 


\section{Figures Captions}

Figure 1. Geometry of periodic topography of wavelength $L$ and amplitude $A$. The $x$-axis is horizontal and the $y$-axis points up. The mean elevation of the topographic surface is at $y=0$. The $n$-axis is normal to the surface and the $p$-axis parallels the surface. The slope angle is given by $\beta$. The stress components shown are positive. The inset figure shows the bell-shaped topography of Savage et $\mathrm{al}^{5}$ defined by the topographic relief $(b)$ and a width parameter $(a)$, with inflection points at $x= \pm(a+b / 2), y=b / 2$.

Figure 2. Contour plots of normalized stress components and stress trajectories below cosinusoidal topography subject just to gravity, for various $A / L$ ratios (in rows): $\sigma_{1} / \rho g A$ (left column); $\sigma_{2} / \rho g A$ (center column); and trajectories perpendicular to the most tensile stress (right column). First row: $A / L=0.02$. Second row: $A / L=0.03$. Third row: $A / L=0.04$. Solutions are from first-order analytical approximations.

Figure 3. Contour plots of normalized stress components and stress trajectories below cosinusoidal topography subject just to gravity, for various $A / L$ ratios (in rows): $\sigma_{1} / \rho g A$ (left column); $\sigma_{2} / \rho g A$ (center column); and trajectories perpendicular to the most tensile stress (right column). First row: $A / L=0.02$. Second row: $A / L=0.03$. Third row: $A / L=0.04$. Solutions are determined using the boundary element method.

Figure 4. Normalized stresses along the topographic surface for various $A / L$ ratios due to gravity alone. (a) $A / L=0.02$. (b) $A / L=0.03$. $A / L=0.04$. A ridge crest corresponds to $x / L=0$, with valley bottoms at $x / L= \pm 0.5$.

Figure 5. Contour plots of normalized stress components and stress trajectories for a uniform compressive horizontal tectonic stress $(T)$, with no gravitational contribution, for various $A / L$ ratios (in rows): $\sigma_{1} /|T|$ (left column); $\sigma_{2} /|T|$ (center column); and trajectories perpendicular to the most tensile stress (right column). First row: $A / L=0.02$. Second row: $A / L=0.03$. Third row: $A / L=0.04$. Solutions are from first-order analytical approximations. The lenticular area 
atop the ridge where $\sigma_{1} /|T|$ is shown as compressive (negative) results from inaccuracy in the approximation.

Figure 6. Contour plots of normalized stress components and stress trajectories for a uniform compressive horizontal tectonic stress $(T)$, with no gravitational contribution, for various $A / L$ ratios (in rows): $\sigma_{1} /|T|$ (left column); $\sigma_{2} /|T|$ (center column); and trajectories perpendicular to the most tensile stress (right column). First row: $A / L=0.02$. Second row: $A / L=0.03$. Third row: $A / L=0.04$. Solutions are determined using the boundary element method.

Figure 7. Normalized stresses along the topographic surface for various $A / L$ ratios due to a uniform horizontal regional stress (T) alone. (a) $A / L=0.02$. (b) $A / L=0.03$. $A / L=0.04$. A ridge crest corresponds to $x / L=0$, with valley bottoms at $x / L= \pm 0.5$.

Figure 8. Contour plots of normalized stress components and stress trajectories for combined gravitational and tectonic stresses, where $T=-0.1 \rho g A$, for various $A / L$ ratios (in rows): $\sigma_{1} / \rho g A$ (left column); $\sigma_{2} / \rho g A$ (center column); and stress trajectories (right column). First row: $A / L=$ 0.02. Second row: $A / L=0.03$. Third row: $A / L=0.04$. Solutions are from first-order analytical approximations.

Figure 9. Contour plots of stress components for combined gravitational and tectonic stresses, where $T=-1 \rho g A$, and stress trajectories for various $A / L$ ratios (in rows): $\sigma_{1} / \rho g A$ (left column); $\sigma_{2} / \rho g A$ (center column); and stress trajectories (right column). First row: $A / L=0.02$. Second row: $A / L=0.03$. Third row: $A / L=0.04$. Solutions are from first-order analytical approximations.

Figure 10. Contour plots of stress components for combined gravitational and tectonic stresses, where $T=-10 \rho g A$, and stress trajectories for various $A / L$ ratios (in rows): $\sigma_{1} / \rho g A$ (left column); $\sigma_{2} / \rho g A$ (center column); and stress trajectories (right column). First row: $A / L=0.02$. Second row: $A / L=0.03$. Third row: $A / L=0.04$. Solutions are from first-order analytical approximations. 
Figure 11. Contour plots of stress components for combined gravitational and tectonic stresses, where $T=-100 \rho g A$, and stress trajectories for various $A / L$ ratios (in rows): $\sigma_{1} / \rho g A$ (left column); $\sigma_{2} / \rho g A$ (center column); and stress trajectories (right column). First row: $A / L=0.02$. Second row: $A / L=0.03$. Third row: $A / L=0.04$. Solutions are from first-order analytical approximations. A lenticular area atop the ridge where $\sigma_{1} /|T|$ is shown as compressive (negative) results from inaccuracy in the approximation; boundary element solutions show that $\sigma_{1} /|T|$ is tensile just below the ridge crest for each value of $A / L$ treated here.

Figure 12. Contour plots of failure potential $(\Phi)$ for combined gravitational and tectonic stresses for $A / L=0.03$. (a) $T=-0.1 \rho g A$. (b) $T=-1 \rho g A$. (c) $T=-10 \rho g A$. (d) $T=-100 \rho g A$. Solutions are from first-order analytical approximations. 
Figure1

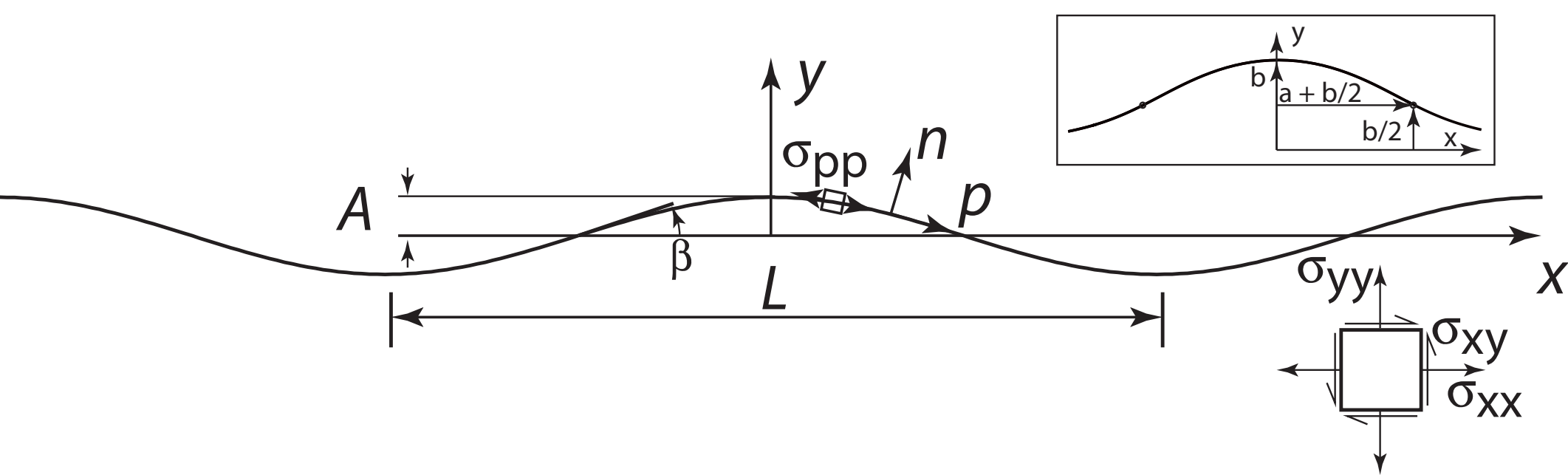


Figure 2
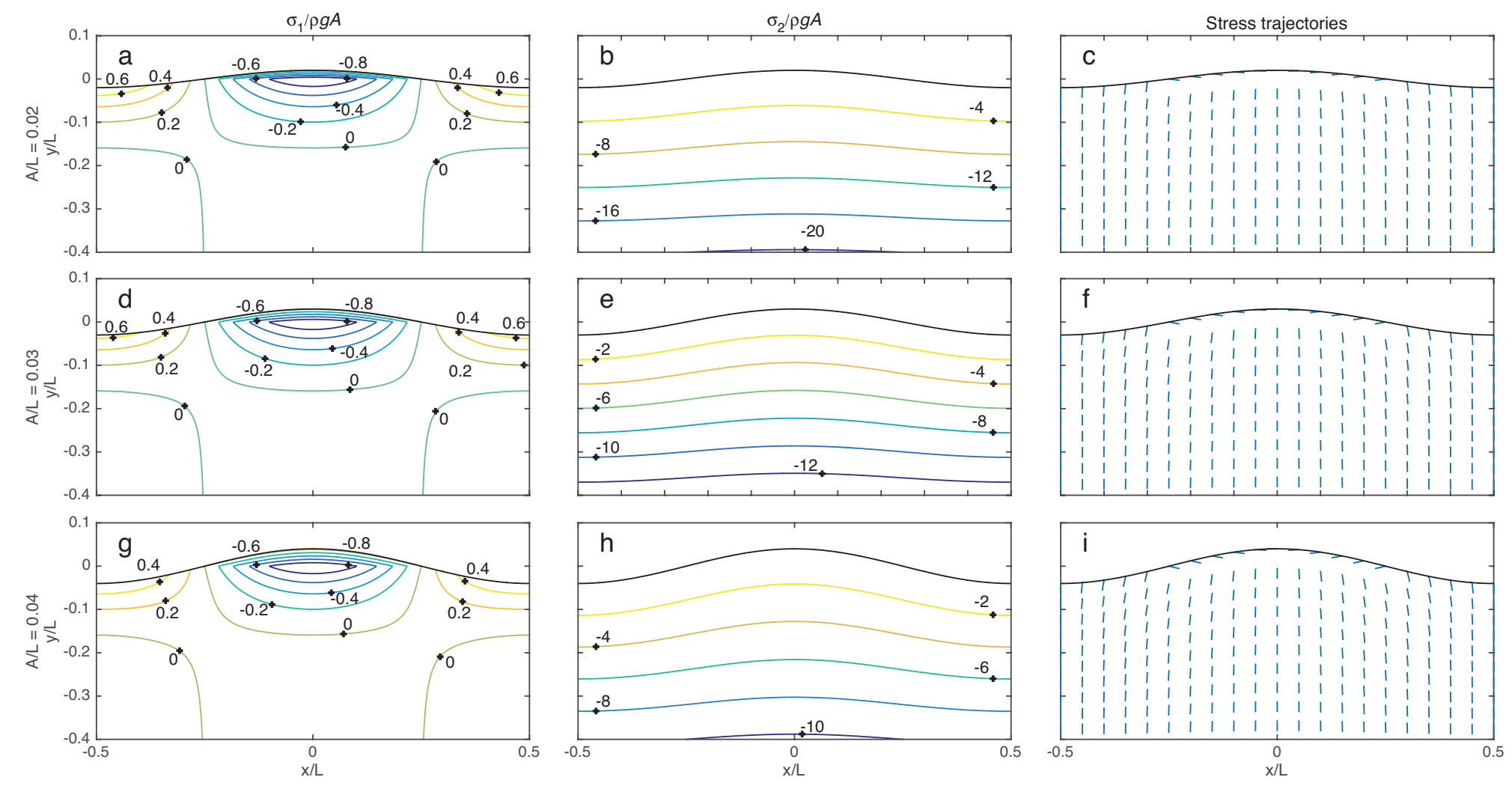
Figure 3
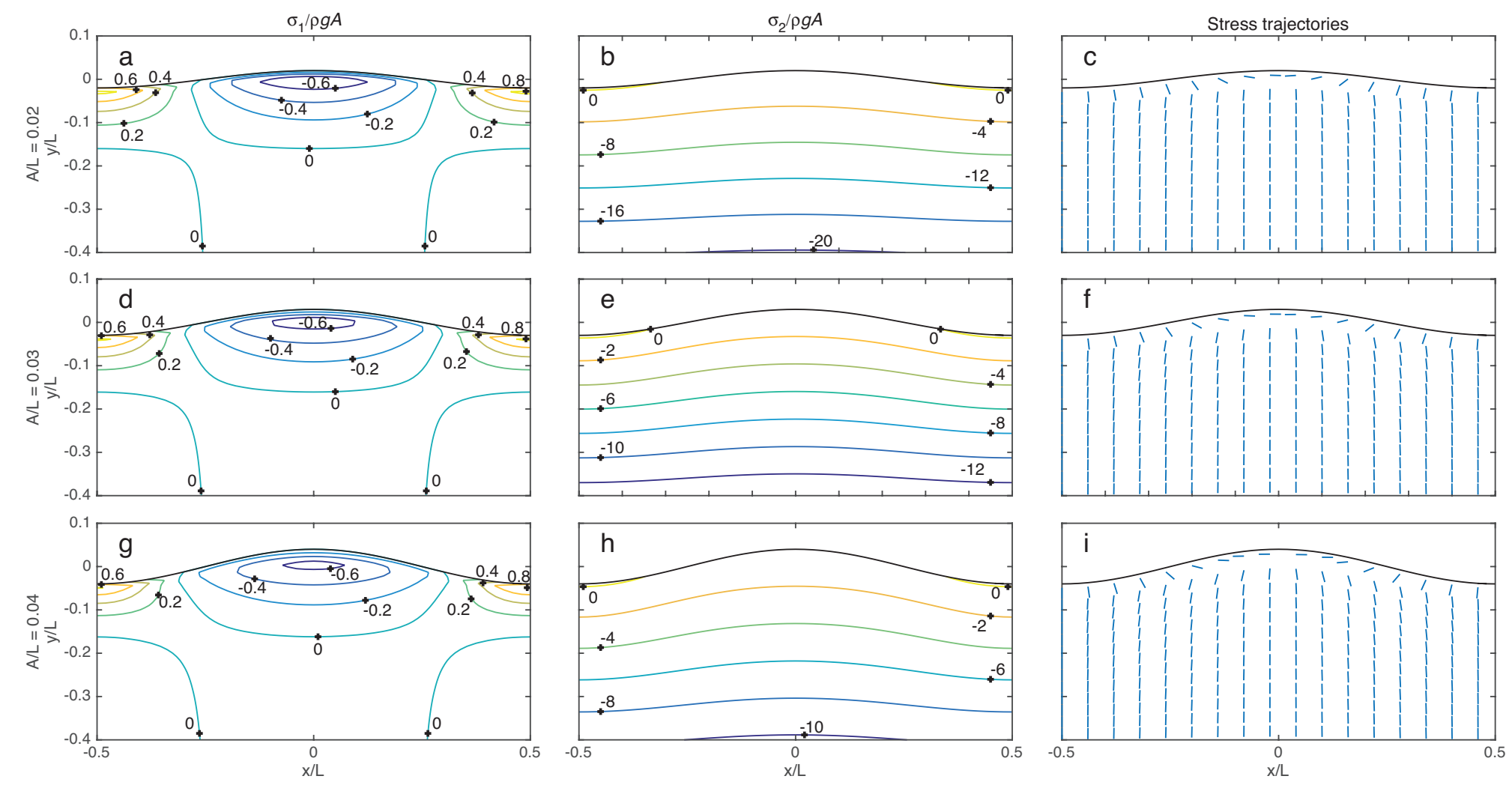

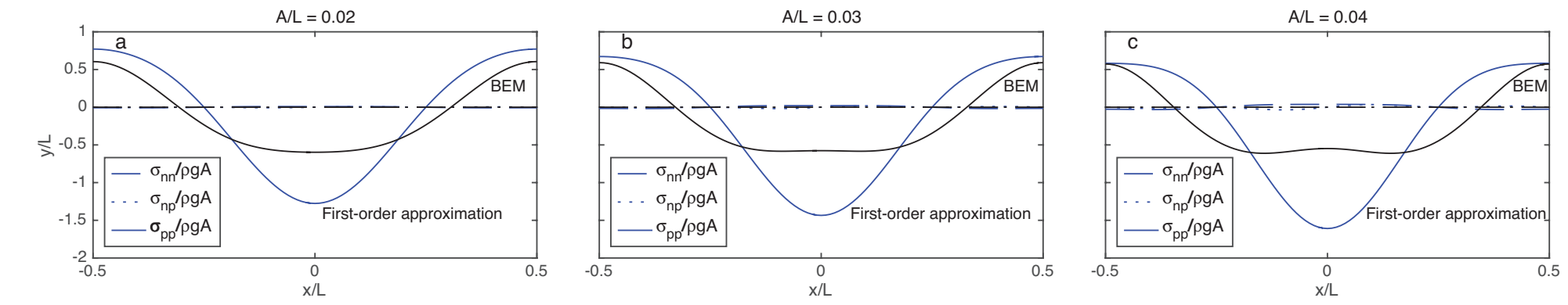

Figure 4 
Figure 5
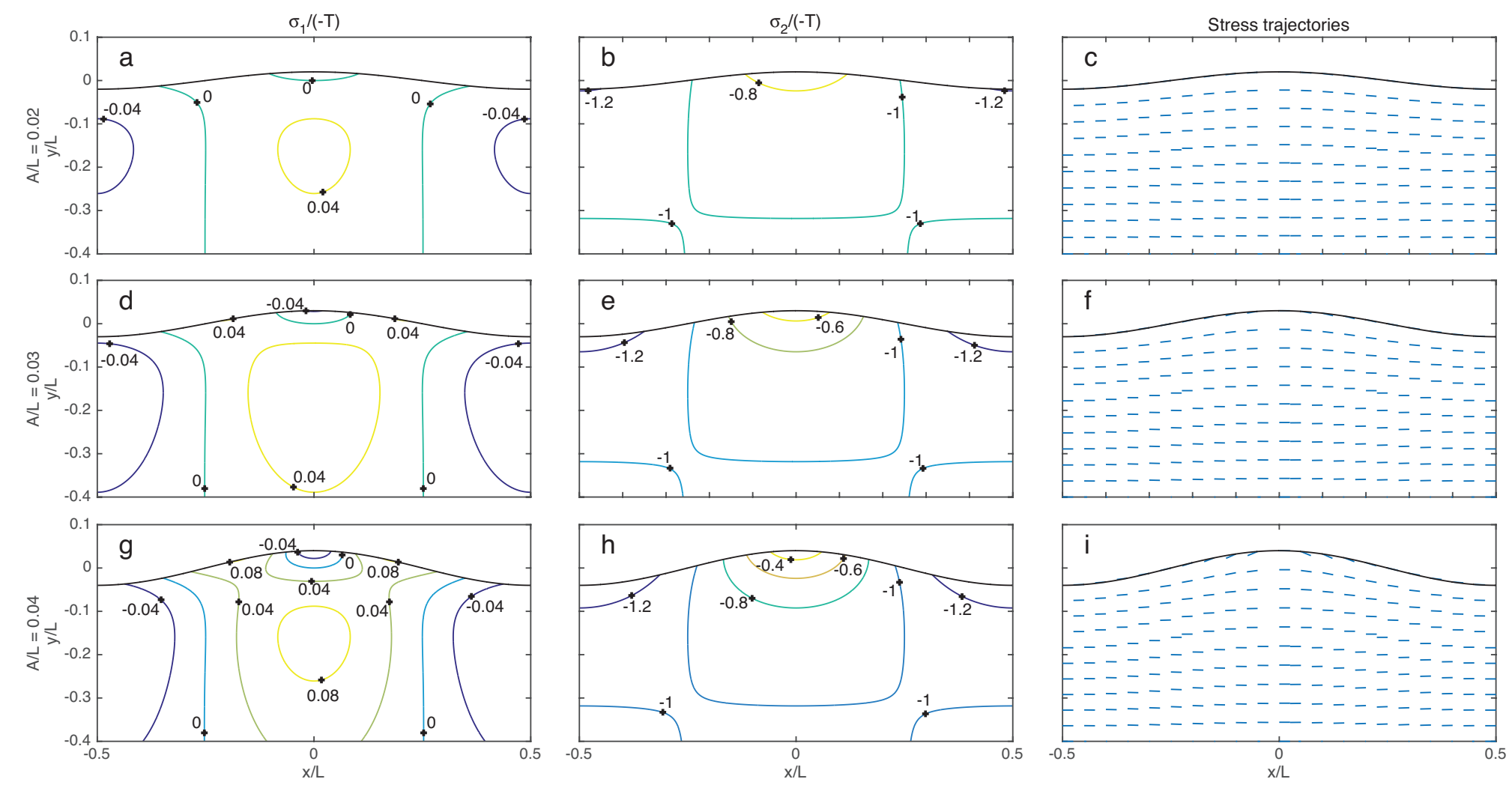

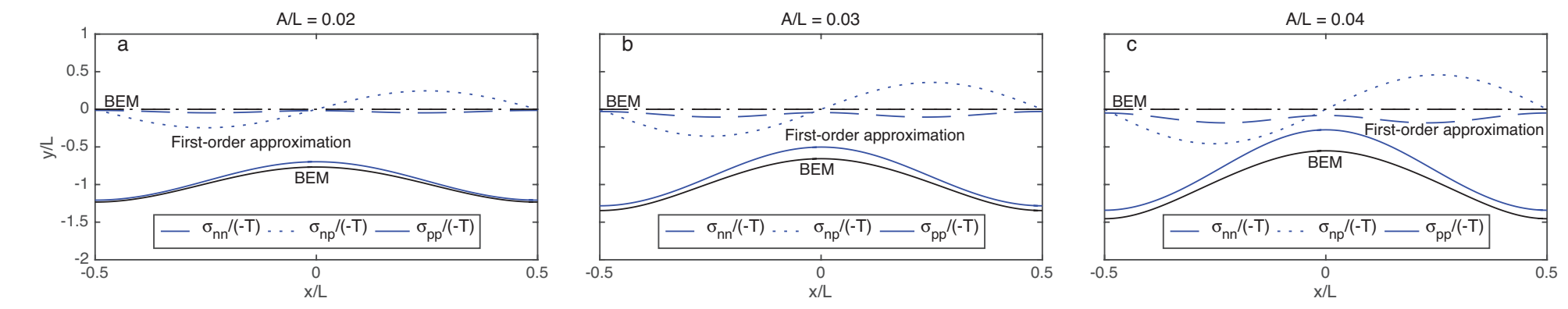

Figure 7 
Figure 8
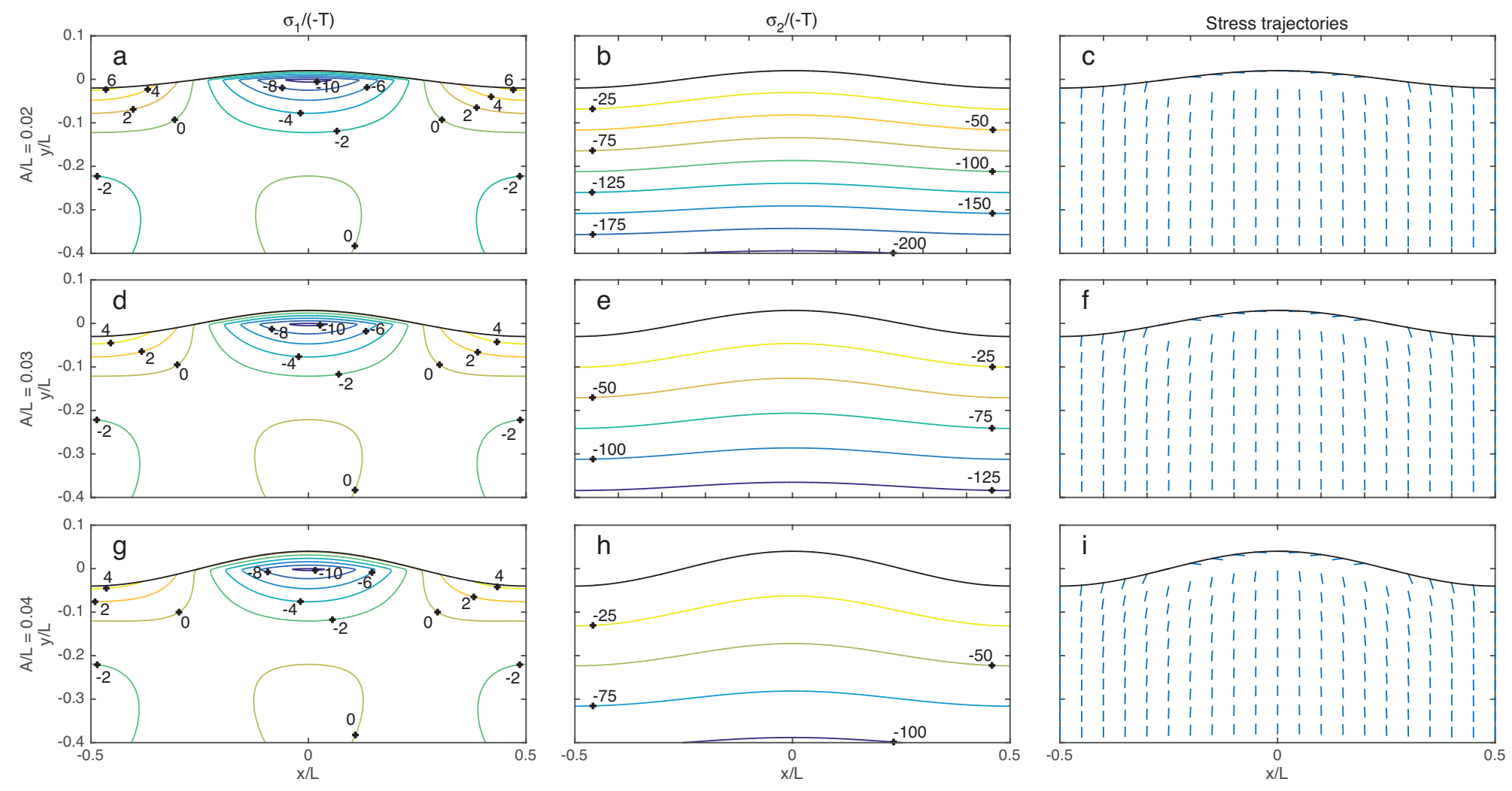
Figure 9
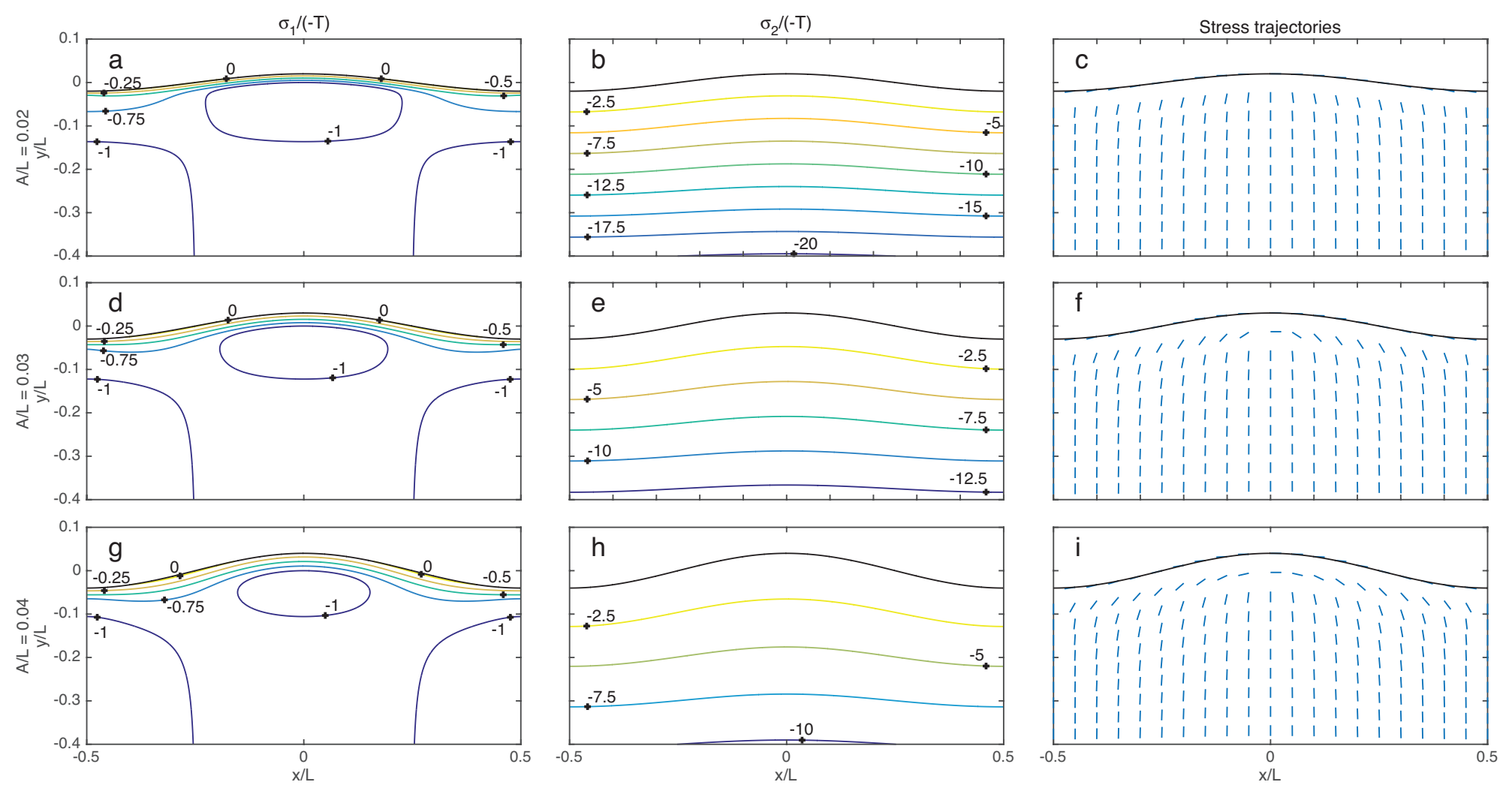

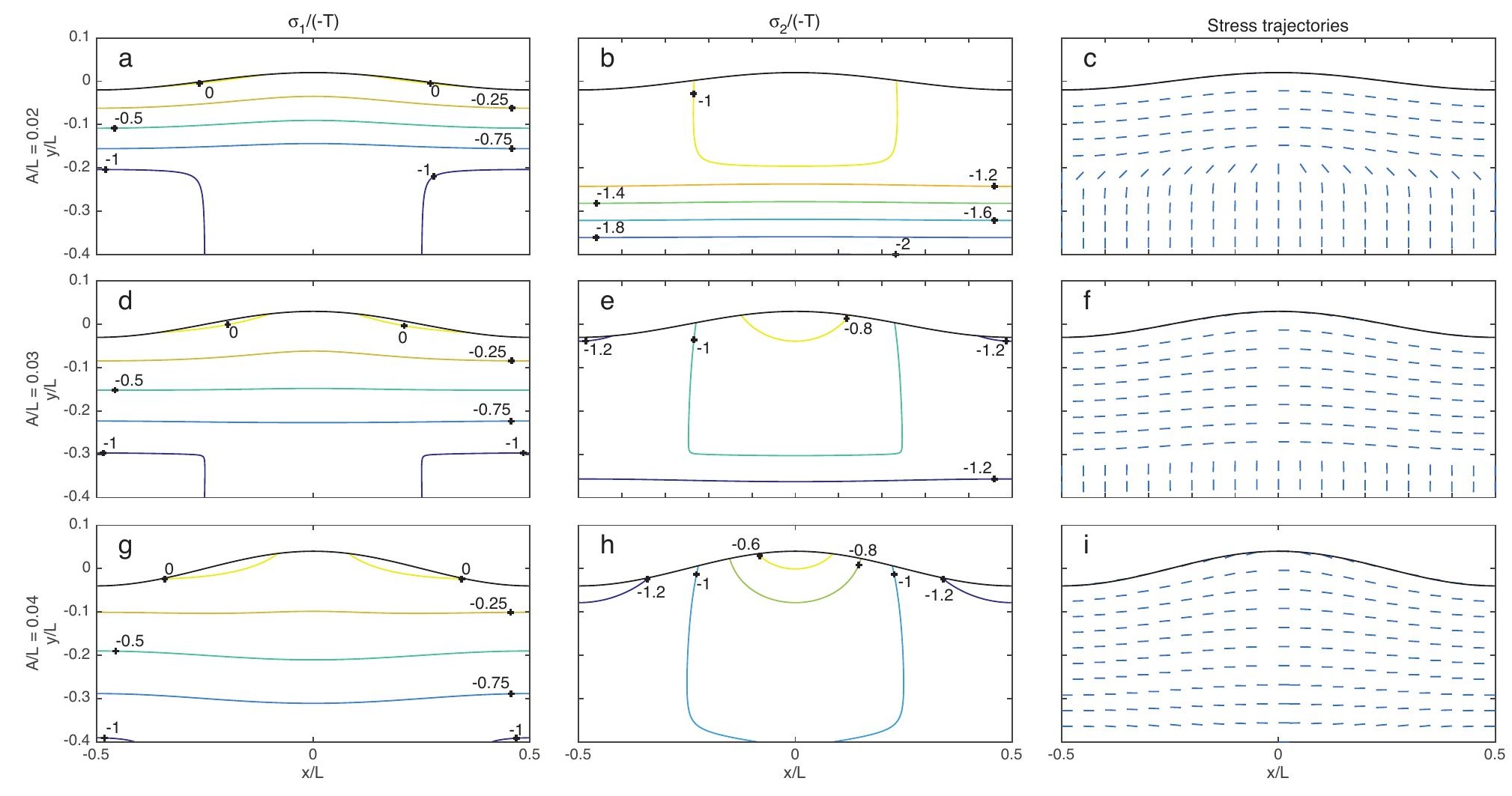

Figure 10

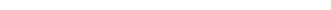

$-$

5


Figure 11
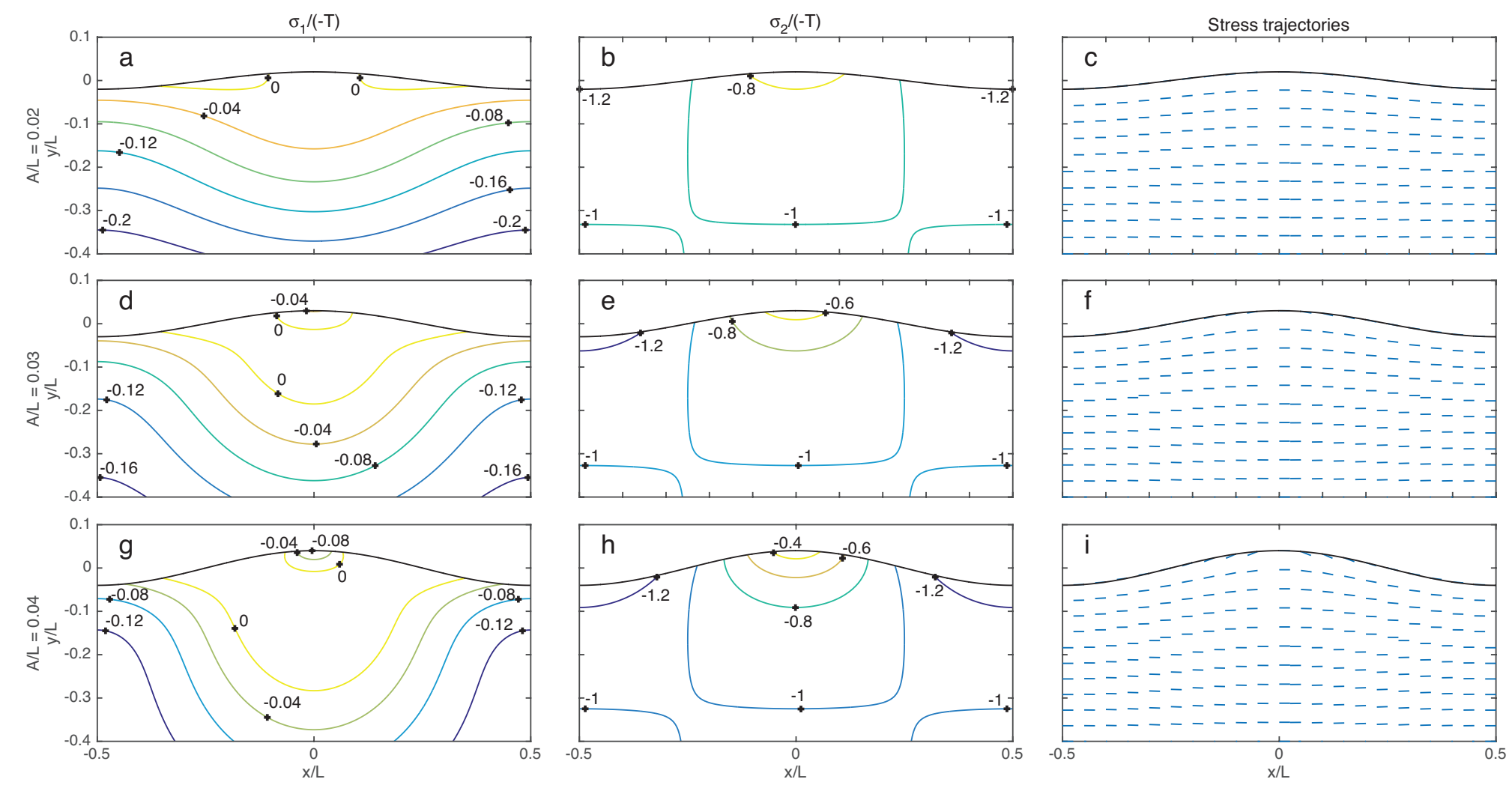
